\title{
Let-7d increases ovarian cancer cell sensitivity to a genistein analog by targeting c-Myc
}

\author{
Ying-Xia Ning ${ }^{1,2, *}$, Xin Luo ${ }^{2}$, Meng $\mathrm{Xu}^{2, *}, \mathrm{Xin} \mathrm{Feng}^{3, *}$ and Jian Wang ${ }^{4}$ \\ ${ }^{1}$ Department of Gynaecology and Obstetrics, The First Affiliated Hospital of Guangzhou Medical University, Guangzhou \\ 510120, China \\ ${ }^{2}$ The First Affiliated Hospital of Jinan University, Guangzhou 510632, China \\ ${ }^{3}$ Cancer Center, Traditional Chinese Medicine-Integrated Hospital, Southern Medical University, Guangzhou 510315, China \\ ${ }^{4}$ Institute of Reproductive and Stem Cell Engineering, Central South University, National Engineering and Research Center \\ of Human Stem Cell, Changsha, 41007, China \\ *These authors have contributed equally to this work \\ Correspondence to: Jian Wang, email: 205106@csu.edu.cn \\ Xin Luo, email: Hluox@126.com \\ Keywords: ovarian cancer, c-Myc, let-7d, genistein analogue, PI3K/AKT
}

Received: October 26, $2016 \quad$ Accepted: June 02, 2017 Published: August 23, 2017

Copyright: Ning et al. This is an open-access article distributed under the terms of the Creative Commons Attribution License 3.0 (CC BY 3.0), which permits unrestricted use, distribution, and reproduction in any medium, provided the original author and source are credited.

\section{ABSTRACT}

c-Myc is a key oncogenic transcription factor that participates in tumor pathogenesis. In this study, we found that levels of c-Myc mRNA and protein were higher in early ovarian cancer tissues than normal ovary samples. Increased c-Myc levels correlated positively with clinical stage I $(\mathrm{Ia}+\mathrm{b} / \mathrm{Ic})$ in ovarian cancer patients. Patients with higher nuclear c-Myc expression had shorter overall survival times than patients with low c-Myc expression. Knocking down c-Myc sensitized ovarian cancer cells to 7-difluoromethoxyl5,4'-di-n-octylgenistein (DFOG), a novel synthetic genistein analogue that suppressed PI3K/AKT signaling in vitro and in vivo. Finally, c-Myc was confirmed to be a direct target of let-7d, and let-7d-induced suppression of c-Myc increased the DFOG-sensitivity of ovarian cancer cells. These results indicate that nuclear c-Myc expression is an unfavorable factor in early ovarian cancer, and that let-7d increases ovarian cancer cell sensitivity to DFOG by suppressing c-Myc and PI3K/AKT signaling.

\section{INTRODUCTION}

Ovarian cancer accounts for only about $3 \%$ of female malignancies, but causes more deaths than any other reproductive system cancer. The pathogenesis of ovarian cancer is complex and driven by dysregulation and aberrant activity of a variety of genes and signaling pathways [1,2]. c-Myc encodes an oncogenic transcription factor that promotes cellular proliferation, migration, invasion, differentiation, and tumor stemness. It is a significant contributor to tumor pathogenesis, and elevated c-Myc expression is an unfavorable factor in many tumor types, including gastric [3], colorectal [4], prostate [5], breast [6], and ovarian [7].
Genistein (GEN) is a natural isoflavone in fruits, nuts, soybeans, and soy-based products. It induces cell apoptosis and cell cycle arrest in various cancer cells [8-10]. DFOG (7-difluoromethoxyl-5,4'-di-n-octylgenistein) is a novel synthetic genistein analogue that exhibits potent anticancer activity. In previous studies, we observed that DFOG markedly suppressed tumor stemness and promoted ovarian cancer cell apoptosis by downregulating FOXM1 and inducing FOXO3a [11-13].

Let-7 family miRNAs, including let-7d, reportedly suppress tumor pathogenesis [14-16]. On the other hand, Let-7d has been observed to contribute to the pathogenesis of some tumors. For example, Di et al observed that let-7d exerts both anti-oncogenic and oncogenic effects in osteosarcoma-derived 3AB-OS 
Table 1: Increased nuclear expression of c-Myc protein in ovarian cancer

\begin{tabular}{lcccc}
\hline & \multicolumn{4}{c}{ Nuclear c-Myc expression } \\
\cline { 2 - 5 } & N & Positive & Negative & P value \\
\hline Ovarian cancer & 138 & 40 & 98 & 0.031 \\
Ovarian tissues & 47 & 6 & 41 & 0 \\
\hline
\end{tabular}

cancer stem cells [17]. In addition, let-7d reportedly directly targets HMGA2 [18], COL3A1, CCL7 and PBX3 [19-23], and thus suppresses tumor cell growth, migration, invasion, and metastasis. In this report, we describe our finding that elevated c-Myc is an unfavorable factor which promotes ovarian cancer pathogenesis, and that suppression of c-Myc by let$7 \mathrm{~d}$ markedly sensitizes cancer cells to DFOG by downregulating the PI3K/AKT pathway.

\section{RESULTS}

\section{c-Myc mRNA is upregulated in ovarian cancer}

When we examined c-Myc mRNA levels using realtime PCR, we observed that c-Myc transcription was significantly higher $(p=0.009)$ in ovarian cancer tissues than normal ovarian tissues (Figure 1A). Moreover, nuclear staining in clinical samples showed that c-Myc expression at the protein level was also higher $(p=0.031)$ in ovarian cancer than normal ovarian tissues (Figure 1B)
(Table 1). These results suggest c-Myc likely plays a key role in the pathogenesis of ovarian cancer.

\section{Correlation between clinicopathological features and nuclear c-Myc expression in stage I ovarian cancer}

The correlation between nuclear c-Myc expression and clinical characteristics was also analyzed. As shown in Table 2, nuclear c-Myc expression did not correlate significantly with patient's age, pathology classification, or grade degree in 138 stage I ovarian cancer cases $(p>0.05)$. However, c-Myc expression correlated positively with clinical stage $(\mathrm{Ia}+\mathrm{b}$ vs. Ic) $(\mathrm{p}=0.015)$.

\section{Nuclear expression of c-Myc correlates negatively with overall survival time in stage I ovarian cancer patients}

Kaplan-Meier analysis with the log-rank test was used to evaluate the prognostic value of c-Myc expression
A

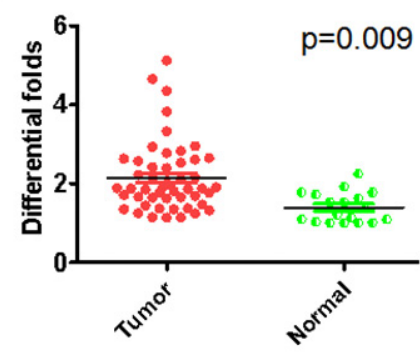

B

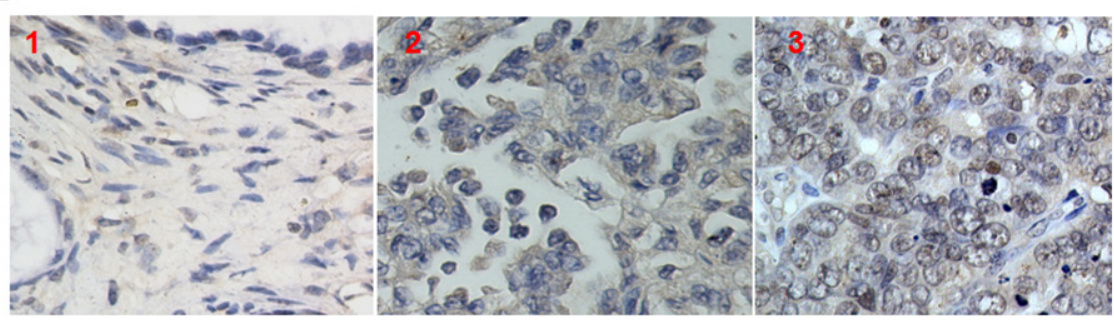

Figure 1: Elevated nuclear c-Myc expression is an unfavorable factor for ovarian cancer patients. (A) c-Myc mRNA levels are downregulated in ovarian cancers as compared to normal ovarian tissues. (B) c-Myc protein expression in ovarian cancer and ovarian tissues. 1. Negative expression of c-Myc in normal ovarian; 2. Positive expression of c-Myc in the cytoplasm of ovarian cancer cells. 3. Positive expression of c-Myc in the nucleus of ovarian cancer cells. (C) Elevated nuclear c-Myc expression is an unfavorable factor for ovarian cancer patients. 
Table 2: The correlation of Nuclear expression of c-Myc with clinical features in ovarian cancer

\begin{tabular}{|c|c|c|c|c|}
\hline \multirow[t]{2}{*}{ Clinical parameter } & \multicolumn{4}{|c|}{ The expression of c-Myc } \\
\hline & $n$ & $\begin{array}{c}\text { Positive expression } \\
\text { (n) }\end{array}$ & $\begin{array}{c}\text { Negative expression } \\
\text { (n) }\end{array}$ & $P$ value \\
\hline \multicolumn{5}{|l|}{ age (year) } \\
\hline$\geqq 50$ & 58 & 17 & 41 & 1.0 \\
\hline$<50$ & 80 & 23 & 57 & \\
\hline \multicolumn{5}{|c|}{ Pathology classification } \\
\hline $\mathrm{S}$ & 107 & 34 & 73 & 0.261 \\
\hline M & 31 & 6 & 25 & \\
\hline \multicolumn{5}{|l|}{ Grade } \\
\hline Low & 44 & 8 & 36 & \\
\hline Middle & 64 & 20 & 44 & 0.111 \\
\hline High & 30 & 12 & 18 & \\
\hline \multicolumn{5}{|l|}{ Clinical stage } \\
\hline $\mathrm{Ia}+\mathrm{b}$ & 112 & 27 & 85 & 0.015 \\
\hline Ic & 26 & 13 & 13 & \\
\hline
\end{tabular}

*Kruskal-Wallis test

for stage I ovarian cancer patients. We observed that c-Myc expression correlated negatively with overall survival time. Patients who were positive for nuclear c-Myc expression had worse prognoses than those who were negative for c-Myc expression (Figure 1C) $(\mathrm{p}<0.001)$.
Stable c-Myc knockdown suppresses pPI3K/ AKT

Stable c-Myc knockdown in ovarian cancer cells was carried out using a lentiviral vector harboring shRNA-c-Myc. Real-time PCR analysis confirmed
A

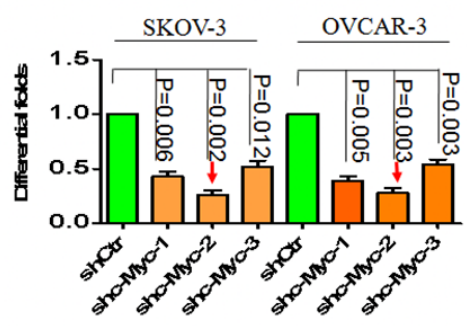

C

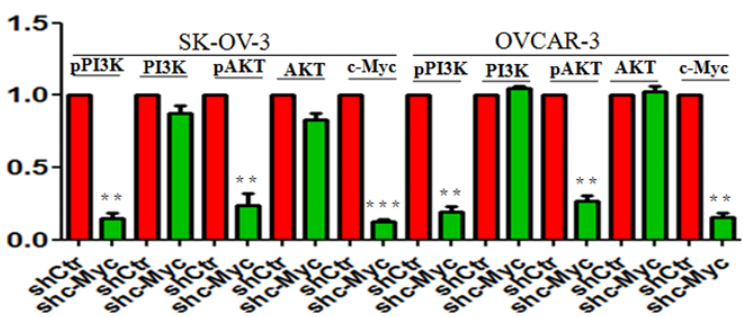

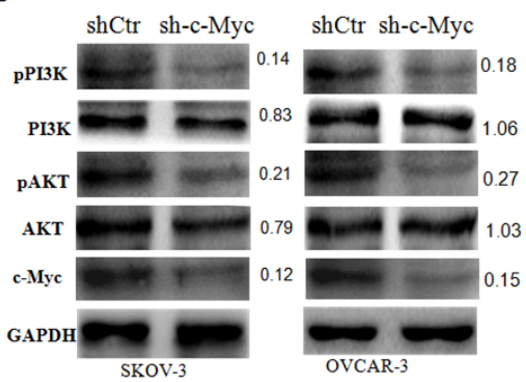

Figure 2: c-Myc knockdown suppresses pPI3K/AKT signaling in ovarian cancer. (A) shRNAs were used to suppress c-Myc mRNA levels in ovarian cancer cells. (B) Suppression of c-Myc blocked pPI3K/AKT signaling, but not total PI3K/AKT protein levels. (C) Bar graph indicating the efficient suppression of pPI3K/AKT signaling after c-Myc knockdown. ${ }^{*} \mathrm{p}<0.05 ; * * \mathrm{p}<0.01 ; * * * \mathrm{p}<0.001$; 
that c-Myc mRNA expression was suppressed in shRNA-c-Myc-2-expressing SKOV3 and OVCAR cells (Figure 2A). A corresponding decrease in c-Myc protein levels was confirmed by western blotting (Figure 3B). Interestingly, we observed that pPI3K/AKT signaling was significantly suppressed following c-Myc knockdown (Figure 2B, 2C).

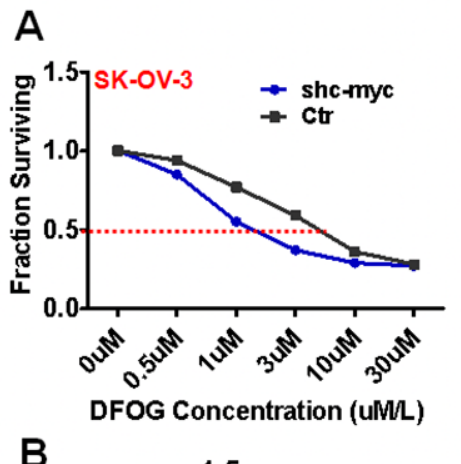

B

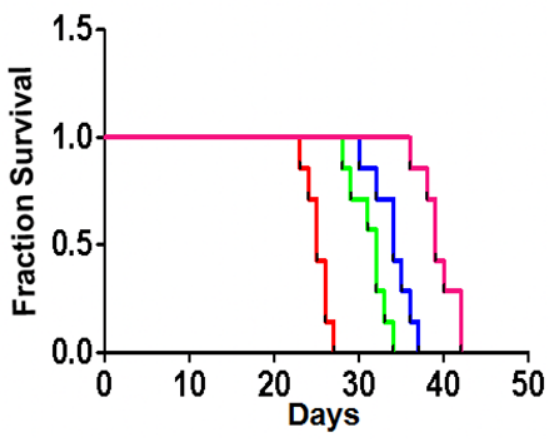

Figure 3: Suppression of c-Myc sensitizes ovarian cancer cells to DFOG in vitro and in vivo. (A and B) Knocking down c-Myc induced ovarian cancer cell sensitivity to DFOG in vitro. (C) Suppression of c-Myc significantly sensitized ovarian cancer cells to DFOG in vivo.

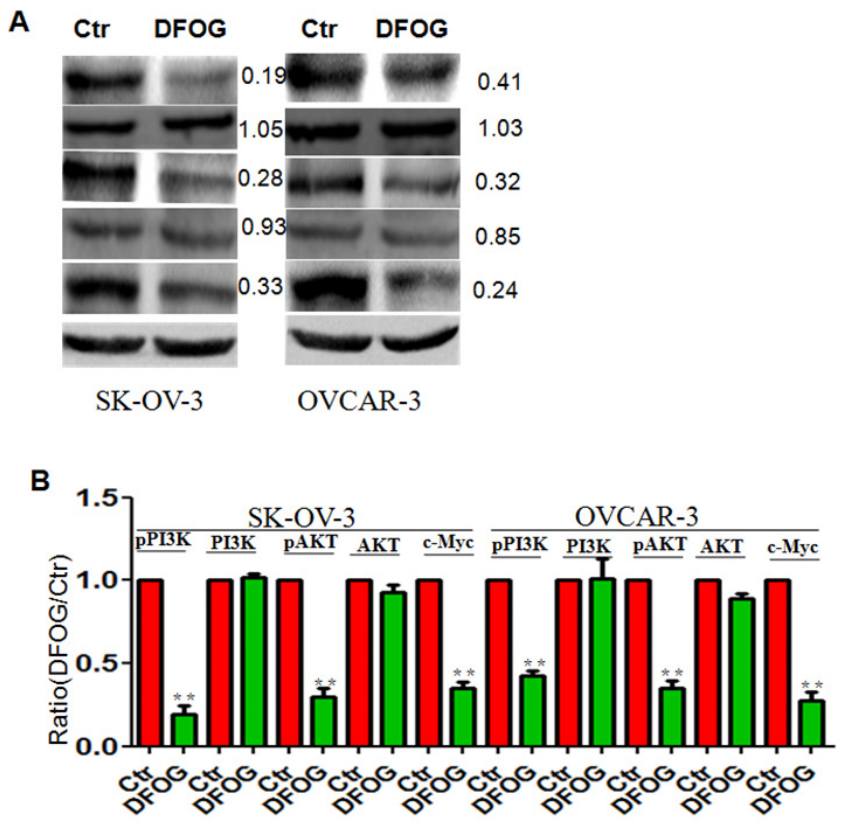

Figure 4: DFOG inhibits pPI3K/AKT and c-Myc signaling in ovarian cancer. (A) pPI3K/AKT and c-Myc signaling were suppressed by DFOG. (B) Bar graph showing the efficient suppression of pPI3K/AKT and c-Myc signaling by DFOG. ${ }^{*} \mathrm{p}<0.05 ; * * \mathrm{p}<0.01$; 
cells to DFOG. The IC50 of shMyc-expressing SKOV3 and OVCAR3 cells was significantly lower than that of their respective control parental lines (SKOV3: $1.12 \pm$ $0.06 \mu \mathrm{m}$ vs $6.11 \pm 0.20 \mu \mathrm{m}, \mathrm{p}=0.003$; OVCAR3: $1.56 \pm$ $0.09 \mu \mathrm{m}$ vs $5.75 \pm 0.29 \mu \mathrm{m}, \mathrm{p}<0.001$ ) (Figure $3 \mathrm{~A}$ ). This sensitizing effect was then examined in vivo with tumors originating from inoculated SKOV3 cells. Survival times for SKOV3 cell tumor-bearing nude mice were prolonged by DFOG treatment. Also exhibiting longer survival times were mice inoculated with c-Myc knockdown cells. And mice administered both c-Myc knockdown cells and DFOG treatment had the longest survival times (Figure 3B) $(P<0.001)$. The average survival times for mice in control, shMyc, control+DFOG and shMyc+DFOG groups were $25.1,34,32.1$ and 45.6 days, respectively.

\section{DFOG suppresses pPI3K/AKT and c-Myc signaling}

Using DFOG to treat ovarian cells, we observed that levels of $\mathrm{pPI} 3 \mathrm{~K} / \mathrm{AKT}$ pathway components were significantly reduced in parental SKOV3 and OVCAR cells. In addition, c-Myc was downregulated after DFOG treatment (Figure 4A, 4B).

\section{Let-7d induces DFOG sensitivity by directly targeting c-Myc}

Let-7d mimics decreased c-Myc and pPI3K/AKT signals in ovarian cancer cells (Figure 5A, 5B), while Let-7d inhibitor exerted the opposite effects (Figure $5 C, 5 D)$. Luciferase activity indicated that c-Myc was a direct target of let-7d (Figure 5E). Moreover, let-7d mimics increased the sensitivity of ovarian cancer cells to DFOG, as evidenced by the lower IC50 levels (SKOV3: $1.12 \pm 0.19 \mu \mathrm{m}$ vs $5.80 \pm 0.35 \mu \mathrm{m}, \mathrm{p}<0.001$; OVCAR3: $2.31 \pm 0.29 \mu \mathrm{m}$ vs $8.52 \pm 0.57 \mu \mathrm{m}, \mathrm{p}<0.001$ ) (Figure $5 \mathrm{~F}$ ). Finally, we observed that blocking let-7d using its inhibitor increased the resistance to DFOG in shc-Mycexpressing ovarian cancer cells (SKOV3: $2.44 \pm 0.6 \mu \mathrm{m}$ vs $8.92 \pm 0.55 \mu \mathrm{m}, \mathrm{p}<0.001$; OVCAR3: $2.23 \pm 0.18 \mu \mathrm{m}$ vs $9.43 \pm 0.39 \mu \mathrm{m}, \mathrm{p}<0.001$ ) (Figure $5 \mathrm{G}$ ).

\section{Let-7d is downregulated and negatively correlates with c-Myc expression}

Real-time PCR assays indicated that let-7d expression was lower in ovarian cancer tissues than normal ovarian tissues $(\mathrm{p}<0.0001)$ (Figure 6A). Moreover, let-7d

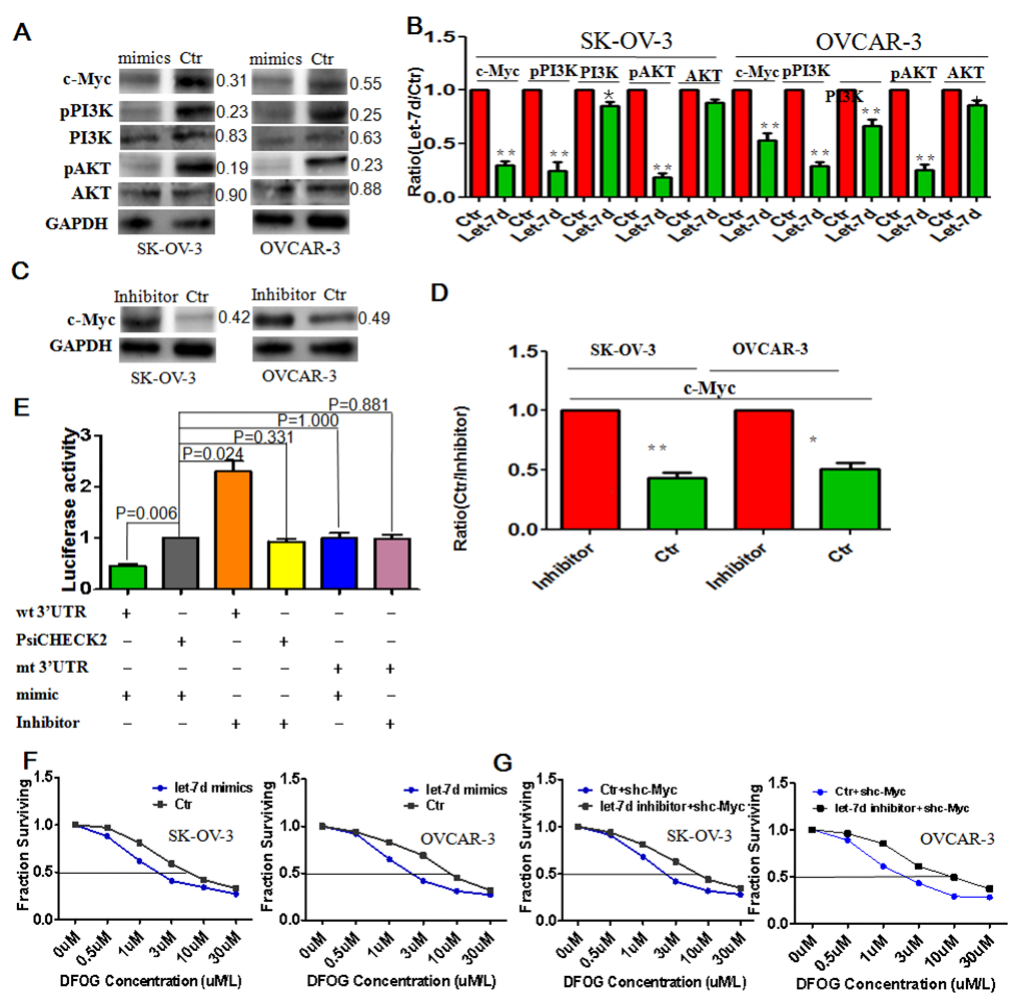

Figure 5: Let-7d directly targets c-Myc to reduce pPI3K/AKT signaling and increase the DFOG sensitivity of ovarian cancer cells. (A) Let-7d suppressed c-Myc and in turn pPI3K/AKT signaling. (B) Bar graph showing the efficient suppression of pPI3K/ AKT and c-Myc signaling by let-7d mimics. (C) Let-7d inhibitor restored expression of c-Myc protein. (D) Bar graph showing the increased efficiency of c-Myc expression induced by let-7d inhibitor. (E) Luciferase activity confirmed c-Myc to be a direct target of let-7d. (F) Let7d induced DFOG sensitivity in ovarian cancer cells. G. Blocking let-7d with its inhibitor increased DFOG resistance in c-Myc knockdown ovarian cancer cells. ${ }^{*} \mathrm{p}<0.05 ; * * \mathrm{p}<0.01$; 
levels correlated negatively with levels of c-Myc mRNA in ovarian cancer tissues (Figure 6B)

\section{DISCUSSION}

c-Myc is a conserved basic helix-loop-helix (HLH) leucine zipper transcription factor that induces cell growth, tumorigenesis, and tumor stemness. In the present study, we firstly observed higher c-Myc mRNA expression in ovarian cancer samples than normal ovarian tissues. This is consistent with an earlier report [7] and is indicative of the importance of c-Myc overexpression in the context of ovarian cancer.

c-Myc activity reportedly leads to dysregulation of numerous genes and thus promotes tumor initiation and progression [24, 25]. When we assessed the differential expression of c-Myc in stage I ovarian cancer and normal ovarian tissue, we found that nuclear expression of c-Myc was markedly higher in the cancer tissue. The finding is consistent with Zeng's observation in prostate cancer [5], and further suggests that c-Myc participates in ovarian carcinogenesis. We also observed that although increased c-Myc did not correlate with age, pathology classification, or grade degree, it was positively related to clinical stage $(\mathrm{Ia}+\mathrm{b}$ vs. Ic). This suggests upregulated c-Myc is also involved in early ovarian cancer progression.

Increased c-Myc expression has been documented as an unfavorable factor in prostate cancer [5]. We also observed that c-Myc correlates negatively with the overall survival time of stage I ovarian cancer patients. Patients exhibiting stronger c-Myc expression had shorter overall survival times than those of patients who lacked c-Myc expression. These data support the idea that c-Myc is a key oncogene in ovarian cancer.

Genistein, 5,7,4'-trihydroxylisoflavone, is a major component of soybean products and reportedly possesses anticancer activities. DFOG is a novel synthetic genistein derivative. We recently reported that DFOG suppresses cell growth and tumor stemness, and that it induces cell apoptosis by suppressing FOXM1 and upregulating FOXO3a in ovarian cancer [11-13]. c-Myc promotes chemotherapeutic resistance in tumors, including ovarian cancer [26-30]. Thus, suppression of c-Myc may be a way to elevate the sensitivity of tumor cells to drugs. Interestingly, we observed that knocking down c-Myc not only markedly elevated ovarian cell sensitivity to DFOG in vitro, but also greatly increased the survival time of tumor-bearing mice.

$\mathrm{PI} 3 \mathrm{~K} / \mathrm{AKT}$ is a classical oncogenic signaling pathway, which promotes tumor pathogenesis and induces chemotherapy resistance [31-33]. Yu et al. observed that endoplasmic reticulum stress promotes autophagy and apoptosis and reverses chemoresistance of human small cell lung cancer cells by inhibiting the PI3K/AKT/ mTOR signal. Zhao et al. found that miR-181c inhibits chemoresistance by suppressing PI3K/AKT in chronic myelocytic leukemia $[34,35]$. In the present study, we observed that knocking down c-Myc expression led to reductions in PI3K/AKT signaling in ovarian cancer cells. This is in contrast to Kaur's report that c-Myc suppresses PI3K/AKT signal in normal cells [36]. However, that observation has not been documented in the context of carcinogenesis and may reflect an inherent difference between normal and malignant cells. Similar to the effect of c-Myc knockdown, we found that DFOG treatment suppressed PI3K/AKT signaling, but also suppressed c-Myc expression. These results provide evidence that c-Myc suppression sensitizes ovarian cells to DFOG treatment.

Let-7d reportedly acts as a tumor suppressor participating in the pathogenesis of some tumors, including ovarian cancer. In previous documents, Let7 (let-7b/c) contributed to HuR-mediated repression of c-Myc expression [37]. In the present study, we found that let-7d directly targets c-Myc and induced PI3K/AKT signaling to increase the DFOG sensitivity of ovarian
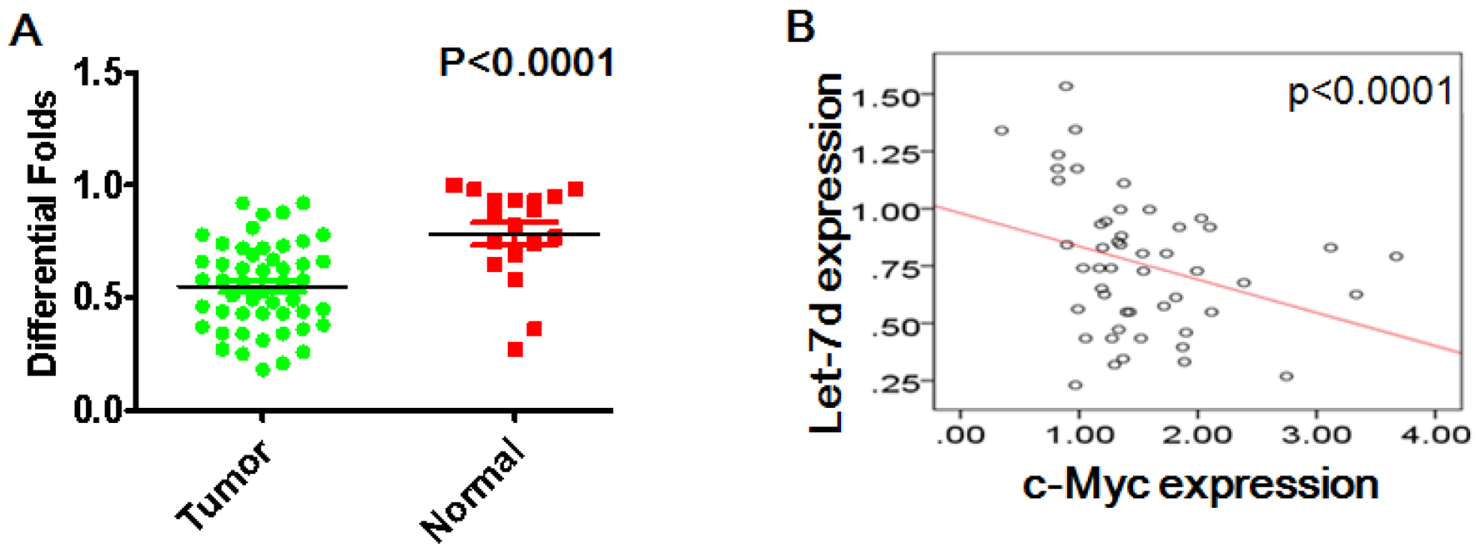

Figure 6: Let-7d levels correlate negatively with c-Myc in ovarian cancer. (A) Let-7d expression was lower in ovarian cancer tissues than normal ovarian tissues. (B) Let-7d levels was correlated negatively with c-Myc expression in ovarian cancer 
cancer cells. We also observed that let-7d expression correlated negatively with c-Myc mRNA expression in ovarian cancers. These data demonstrate the importance of the let-7d/c-Myc signal in ovarian cancer.

In summary, our study has demonstrated that c-Myc mRNA levels are significantly elevated in ovarian cancer tissues. c-Myc expression correlates positively with clinical progression and is predictive of a poor prognosis in stage I ovarian cancer patients. Our data also demonstrate that c-Myc suppression sensitizes ovarian cancer cells to DFOG by inactivating PI3K/AKT signaling. Finally, c-Myc is a direct target of the tumor suppressor let-7d, and its suppression is associated with let-7d-mediated increases in DFOG-sensitivity in ovarian cancer.

\section{MATERIALS AND METHODS}

\section{Cell culture and sample collection}

The SKOV3 and OVCAR ovarian cancer cell lines were purchased from the Shanghai cell bank of the Chinese Academy of Sciences. The cells were maintained in RPMI-1640 medium supplemented with $10 \%$ fetal calf serum (FCS) (PAA Laboratories, Inc, Pasching, Austria). Tissues from patients included 50 fresh ovarian cancer samples and 18 normal ovarian samples. In addition, paraffin-embedded ovarian samples (138 clinical stage I cancers and 47 normal ovary) were obtained from the First Affiliated Hospital of Guangzhou Medical University. Clinical processes were approved by the hospital Ethics Committee, and patients gave informed written consent.

\section{RNA isolation and qRT-PCR}

Total RNA was isolated using TRIzol reagent according to the manufacturer's protocol (Invitrogen, USA), after which $1 \mu \mathrm{g}$ of RNA was synthesized to cDNA using SuperScriptase III (Invitrogen) with random primers. PCR was performed using primers for c-Myc (sense: 5'GAGGAGAATGTCAAGAGGCG3'; antisense: ATAACTACCTTGGGGGCCTT) in accordance with the manufacturer's instructions (Takara, Shiga, Japan). PCR reactions were repeated three times, and $\beta$-actin (sense: TGACGTGGACATCCGCAAAG; antisense: CTGGAAGGTGGACAGCGAGG) was used as an internal control.

\section{Immunohistochemistry}

Immunohistochemistry was carried out as described previously [10] using a rabbit anti-human c-Myc polyclonal antibody (1:100 dilution; Santa Cruz Biotechnology, USA). Sections were stained using DAB, counterstained with hematoxylin, mounted in neutral gum, and visualized using a bright field microscope.

\section{Evaluation of staining}

Stained tissue sections were reviewed separately by two pathologists blinded to the clinical parameters. Samples with $\geq 10 \%$ nuclear staining were considered positive for nuclear expression. Samples with $<10 \%$ staining were considered negative for nuclear expression.

\section{Western blot analysis}

Thirty-microgram aliquots of protein were subjected to $10 \%$ SDS-PAGE, after which the separated proteins were transferred to PVDF membranes. The membranes were then incubated first for $1-2 \mathrm{~h}$ in blocking solution, then for $1 \mathrm{~h}$ with the following primary antibodies: rabbit polyclonal c-Myc, PI3K, pPI3K, AKT, pAKT, and GAPDH (Cell Signaling Technology, Danvers, USA). An HRP-conjugated anti-rabbit IgG antibody was used as the secondary antibody (Zhongshan, Beijing, China). Signals were developed using enhanced chemiluminescence reagents (Pierce, Rockford, IL).

\section{Construction of stable c-Myc knockdown ovarian cancer cell lines}

Four lentiviral vectors, three harboring shMycs and one harboring shCtrl, were prepared by Genechem Incorporation (Shanghai, China). These constructs were used to infect SKOV3 and OVCAR ovarian cancer cells, and polyclonal cells exhibiting GFP positivity were selected for further analysis using FACS.

\section{MTT cytotoxicity assay}

shc-Myc SKOV3 and OVCAR ovarian cancer cells and their respective control cells were seeded into 96-well plates to a density of $5 \times 10^{3}$ cells/well and then incubated in $100 \mu \mathrm{l}$ DMEM supplemented with $10 \%$ FBS. Once the cells attached, $0,0.5,1,3,10$, or $30 \mu \mathrm{M}$ DFOG was added to the wells, which were then incubated for $48 \mathrm{~h}$ at $37^{\circ} \mathrm{C}$ under $5 \% \mathrm{CO}_{2}$. Thereafter, $10 \mu \mathrm{l}$ of MTT $(5 \mathrm{mg} /$ ml) (Sigma, St. Louis, MO, USA) were added to each well, and plates were incubated for an additional $4 \mathrm{~h}$ at $37^{\circ} \mathrm{C}$. The supernatants were then removed, and $100 \mu \mathrm{l}$ of DMSO (Sigma) were added to each well. The optical density (OD) of each well at $490 \mathrm{~nm}$ was then measured. The calculated rates were then used for curve fitting and for calculating the half-maximal inhibitory concentration (IC50). Experiments were repeated three times.

\section{In vivo experiments in nude mice}

In vivo experiments were approved by the Animal Care and Use Committee of Guangzhou Medical University. All mice were provided by the Central Animal Facility of Guangzhou Medical University at 4 weeks of age. Nude mice (BALB/C, nu/nu) were injected 
intraperitoneally with approximately $6 \times 10^{5}$ c-Mycsilenced SKOV3 cells or control cells in $0.2 \mathrm{~mL}$ of buffered saline. Tumors were allowed to grow for 3 days, after which the animals were randomized into four groups (shCtr; shMyc; shCtr+DFOG; shMyc+DFOG), which were administered normal saline (NS) or DFOG $(10 \mathrm{mg}$ / $\mathrm{kg}$ ) every 4 days. Survival was calculated using Kaplan Meier analysis.

\section{Transfection with let-7d mimics and its inhibitor}

let-7d mimic and its inhibitor were purchased from Guangzhou RiboBio (RiboBio Inc, China). Ovarian cancer cells were seeded into a 6-well or 96-well plate (Nest, Biotech, China) to $40 \%$ confluence. After incubation for $24 \mathrm{~h}$, the cells were transfected with let-7d mimics or its inhibitor using TurboFectTM siRNA Transfection.

\section{miRNA target validation}

Based on analysis using miRwalk software (University of Heidelberg, Mannheim, Germany), c-Myc was predicted to be a direct target of let-7d. A 256-base fragment of the wild-type c-Myc 3'UTR with a let-7d binding site was amplified and then cloned into psiCHECK-2 vector (WT) containing XhoI and NotI restriction enzyme sites. Site-directed mutagenesis of the let-7d binding site in c-Myc 3'UTR was performed using a GeneTailor System (Invitrogen), and its cloning into psiCHECK-2 yielded the mutant-type vector MT. For reporter assays, WT, MT or control psiCHECK-2 vector were cotransfected into ovarian cancer cells with let-7d mimics or inhibitor. Luciferase activity was measured $48 \mathrm{~h}$ after transfection using a Dual-Luciferase Reporter Assay System (Promega Corporation, Madison, WI, USA).

\section{Statistical analysis}

All data were analyzed for statistical significance using SPSS 13.0 software and presented as the mean \pm SD. Values of $\mathrm{P}<0.05$ were considered statistically significant. Student's t-test was applied to examine differences in c-Myc mRNA expression between normal and ovarian cancer tissues. One-way ANOVA was used to determine the differences in between groups in all in vitro analyses.

\section{Abbreviations}

DFOG, 7-difluoromethoxyl-5,4'-di-n-octylgenistein; GEN, Genistein; qRT-qPCR, Quantification reverse transcription-quantitative polymerase chain reaction.

\section{Author contributions}

YXN, MX, XF, and XL carried out all the experiments. JW and XL participated the study design. JW interpreted the data, drafted the manuscript and gave final approval of the manuscript.

\section{ACKNOWLEDGMENTS}

This work was supported by National Natural Science Foundation of China (No. 81301894), Guangzhou Science and Information Bureau Item (No.201300000151) of China, Guangdong Province Science and Technique Department Item (No.2014A020211028) of China, the Scientific Research Project for Medical College of Guangzhou City Bureau of Education (No.1201410508) and Guangdong Province Science and Technique Department Item (No.2014A020212428) of China.

\section{CONFLICTS OF INTEREST}

The authors declare that they have no competing interests.

\section{REFERENCES}

1. Trémollieres F, Lopès P, Gompel A. Groupe d'Etude sur la Ménopause et le Vieillissement hormonal: Hormone therapy and ovarian cancer. Lancet. 2015; 386:1038.

2. Kotsopoulos J, Lubinski J, Gronwald J, Cybulski C, Demsky R, Neuhausen SL, Kim-Sing C, Tung N, Friedman S, Senter L, Weitzel J, Karlan B, Moller P, et al. The Hereditary Breast Cancer Clinical Study Group: Factors influencing ovulation and the risk of ovarian cancer in BRCA1 and BRCA2 mutation carriers. Int J Cancer. 2015; 137:1136-46.

3. Stah P, Seeschaaf C, Lebok P, Kutup A, Bockhorn M, Izbicki JR, Bokemeyer C, Simon R, Sauter G, Marx AH. Heterogeneity of amplification of HER2, EGFR, CCND1 and MYC in gastric cancer. BMC Gastroenterol. 2015; 15:7.

4. Kriegl L, Vieth M, Kirchner T, Menssen A. Up-regulation of C-MYC and SIRT1 expression correlates with malignant transformation in the serrated route to colorectal cancer. Oncotarget. 2012; 3:1182-93.https://doi.org/10.18632/ oncotarget.628.

5. Zeng W, Sun H, Meng F, Liu Z, Xiong J, Zhou S, Li F, Hu J, $\mathrm{Hu}$ Z, Liu Z. Nuclear C-MYC expression level is associated with disease progression and potentially predictive of two year overall survival in prostate cancer. Int J Clin Exp Pathol. 2015; 8:1878-88.

6. Ren J, Jin F, Yu Z, Zhao L, Wang L, Bai X, Zhao H, Yao W, Mi X, Wang E, Olopade OI, Wei M. MYC overexpression and poor prognosis in sporadic breast cancer with BRCA1 deficiency. Tumour Biol. 2013; 34:3945-58.

7. Chen CH, Shen J, Lee WJ, Chow SN. Overexpression of cyclin D1 and c-Myc gene products in human primary epithelial ovarian cancer. Int J Gynecol Cancer. 2005; $15: 878-83$. 
8. Roh T, Kim SW, Moon SH, Nam MJ. Genistein induces apoptosis by down-regulating thioredoxin-1 in human hepatocellular carcinoma SNU-449 cells. Food Chem Toxicol. 2016; 97:127-34

9. Yang YM, Yang Y, Dai WW, Li XM, Ma JQ, Tang LP. Genistein-induced apoptosis is mediated by endoplasmic reticulum stress in cervical cancer cells. Eur Rev Med Pharmacol Sci. 2016; 20:3292-6.

10. Tsafa E, Al-Bahrani M, Bentayebi K, Przystal J, Suwan $\mathrm{K}$, Hajitou A. The natural dietary genistein boosts bacteriophage-mediated cancer cell killing by improving phage-targeted tumor cell transduction. Oncotarget. 2016; 7:52135-49. https://doi.org/10.18632/oncotarget.10662.

11. Ning YX, Li QX, Ren KQ, Quan MF, Cao JG. 7-difluoromethoxyl-5,4'-di-n-octyl genistein inhibits ovarian cancer stem cell characteristics through the downregulation of FOXM1. Oncol Lett. 2014; 8:295-300.

12. Ning Y, Luo C, Ren K, Quan M, Cao J. FOXO3a-mediated suppression of the self-renewal capacity of sphere-forming cells derived from the ovarian cancer SKOV3 cell line by 7-difluoromethoxyl-5,4'-di-n-octyl genistein. Mol Med Rep. 2014; 9:1982-8.

13. Ning Y, Li Q, Xiang H, Liu F, Cao J. Apoptosis induced by 7-difluoromethoxyl-5,4'-di-n-octyl genistein via the inactivation of FoxM1 in ovarian cancer cells. Oncol Rep. 2012; 27:1857-64.

14. Powers JT, Tsanov KM, Pearson DS, Roels F, Spina CS, Ebright R, Seligson M, de Soysa Y, Cahan P, Theißen J, Tu HC, Han A, Kurek KC, et al. Multiple mechanisms disrupt the let-7 microRNA family in neuroblastoma. Nature. 2016; 535:246-51.

15. Xie C, Chen W, Zhang M, Cai Q, Xu W, Li X, Jiang S. MDM4 regulation by the let-7 miRNA family in the DNA damage response of glioma cells. FEBS Lett. 2015; 589:1958-65.

16. Lin LT, Chang CY, Chang CH, Wang HE, Chiou SH, Liu RS, Lee TW, Lee YJ. Involvement of let-7 microRNA for the therapeutic effects of Rhenium-188-embedded liposomal nanoparticles on orthotopic human head and neck cancer model. Oncotarget. 2016; 7:65782-65796.https://doi. org/10.18632/oncotarget.11666.

17. Di Fiore R, Drago-Ferrante R, Pentimalli F, Di Marzo D, Forte IM, Carlisi D, De Blasio A, Tesoriere G, Giordano A, Vento R. Let-7d miRNA Shows Both Antioncogenic and Oncogenic Functions in Osteosarcoma-Derived 3AB-OS Cancer Stem Cells. J Cell Physiol. 2016; 231:1832-41.

18. Ye H, Chen J, Huang X, Guo A, Hao P. Construction of let-7d expression vector and its inhibitory effect on HMGA2 and ras expression in human ovarian cancer cells in vitro. [Article in Chinese]. Nan Fang Yi Ke Da Xue Xue Bao. 2012; 32:1752-7.

19. Luan J, Wang J, Su Q, Chen X, Jiang G, Xu X. Metaanalysis of the differentially expressed microRNA profiles in nasopharyngeal carcinoma. Oncotarget. 2016; 7:1051321.https://doi.org/10.18632/oncotarget.7013.

20. Manikandan M, Deva Magendhra Rao AK, Arunkumar G, Manickavasagam M, Rajkumar KS, Rajaraman R, Munirajan AK. Oral squamous cell carcinoma: microRNA expression profiling and integrative analyses for elucidation of tumourigenesis mechanism. Mol Cancer. 2016; 15:28.

21. Nuovo GJ, Garofalo M, Valeri N, Roulstone V, Volinia S, Cohn DE, Phelps M, Harrington KJ, Vile R, Melcher A, Galanis E, Sehl S, Adair R, et al. Reovirus-associated reduction of microRNA-let-7d is related to the increased apoptotic death of cancer cells in clinical samples. Mod Pathol. 2012; 25:1333-44.

22. Su B, Zhao W, Shi B, Zhang Z, Yu X, Xie F, Guo Z, Zhang X, Liu J, Shen Q, Wang J, Li X, Zhang Z, et al. Let-7d suppresses growth, metastasis, and tumor macrophage infiltration in renal cell carcinoma by targeting COL3A1 and CCL7. Mol Cancer. 2014; 13:206.

23. Ramberg H, Alshbib A, Berge V, Svindland A, Taskén KA. Regulation of PBX3 expression by androgen and Let-7d in prostate cancer. Mol Cancer. 2011; 10:50.

24. Boudjadi S, Carrier JC, Groulx JF, Beaulieu JF. Integrin $\alpha 1 \beta 1$ expression is controlled by c-MYC in colorectal cancer cells. Oncogene. 2016; 35:1671-8

25. Kim T, Cui R, Jeon YJ, Fadda P, Alder H, Croce CM. MYCrepressed long noncoding RNAs antagonize MYC-induced cell proliferation and cell cycle progression. Oncotarget. 2015; 6:18780-9. https://doi.org/10.18632/oncotarget.3909.

26. Liu L, Zhang J, Yang X, Fang C, Xu H, Xi X. SALL4 as an Epithelial-Mesenchymal Transition and Drug Resistance Inducer through the Regulation of c-Myc in Endometrial Cancer. PLoS One. 2015; 10:138515.

27. Zhang Y, Chen HX, Zhou SY, Wang SX, Zheng K, Xu DD, Liu YT, Wang XY, Wang X, Yan HZ, Zhang L, Liu QY, Chen WQ, et al. Sp1 and c-Myc modulate drug resistance of leukemia stem cells by regulating survivin expression through the ERK-MSK MAPK signaling pathway. Mol Cancer. 2015; 14:56.

28. Fico A, Alfano D, Valentino A, Vasta V, Cavalcanti E, Travali S, Patriarca EJ, Caputo E. C-Myc modulation: a key role in melanoma drug response. Cancer Biol Ther. 2015; 16:1375-86.

29. Pyndiah S, Tanida S, Ahmed KM, Cassimere EK, Choe C, Sakamuro D. c-MYC suppresses BIN1 to release poly(ADP-ribose) polymerase 1: a mechanism by which cancer cells acquire cisplatin resistance. Sci Signal. 2011; 4:ra19.

30. Reyes-González JM, Armaiz-Peña GN, Mangala LS, Valiyeva F, Ivan C, Pradeep S, Echevarría-Vargas IM, Rivera-Reyes A, Sood AK, Vivas-Mejía PE. Targeting c-MYC in Platinum-Resistant Ovarian Cancer. Mol Cancer Ther. 2015; 14:2260-9.

31. Zhao M, Luo R, Liu Y, Gao L, Fu Z, Fu Q, Luo X, Chen Y, Deng X, Liang Z, Li X, Cheng C, Liu Z, et al. miR-3188 
regulates nasopharyngeal carcinoma proliferation and chemosensitivity through a FOXO1-modulated positive feedback loop with mTOR-p-PI3K/AKT-c-JUN. Nat Commun. 2016; 7:11309.

32. Kang Q, Yan S. Piperlongumine reverses doxorubicin resistance through the PI3K/Akt signaling pathway in K562/A02 human leukemia cells. Exp Ther Med. 2015; 9:1345-1350.

33. Zhen Y, Fang W, Zhao M, Luo R, Liu Y, Fu Q, Chen Y, Cheng C, Zhang Y, Liu Z. miR-374a-CCND1-pPI3K/AKTc-JUN feedback loop modulated by PDCD4 suppresses cell growth, metastasis, and sensitizes nasopharyngeal carcinoma to cisplatin. Oncogene. 2017; 36:275-85.

34. Yu XS, Du J, Fan YJ, Liu FJ, Cao LL, Liang N, Xu DG, Zhang JD. Activation of endoplasmic reticulum stress promotes autophagy and apoptosis and reverses chemoresistance of human small cell lung cancer cells by inhibiting the PI3K/AKT/mTOR signaling pathway. Oncotarget. 2016; 7:76827-39. https://doi.org/10.18632/ oncotarget. 12718

35. Zhao L, Li Y, Song X, Zhou H, Li N, Miao Y, Jia L. Upregulation of miR-181c inhibits chemoresistance by targeting ST8SIA4 in chronic myelocytic leukemia. Oncotarget. 2016; 7:60074-86. https://doi.org/10.18632/ oncotarget.11054

36. Kaur M, Cole MD. MYC acts via the PTEN tumor suppressor to elicit autoregulation and genome-wide gene repression by activation of the Ezh2 methyltransferase. Cancer Res. 2013; 73:695-705.

37. Kim HH, Kuwano Y, Srikantan S, Lee EK, Martindale JL, Gorospe M. HuR recruits let-7/RISC to repress c-Myc expression. Genes Dev. 2009; 23:1743-8. 